\title{
Clinical Evaluation of Maxillary Anterior Teeth in Relation to Golden Proportion, Red Proportion and Golden Percentage
}

Anjana Maharjan, ${ }^{1}$ Sarita Joshi

${ }^{1}$ National Academy of Medical Sciences, Bir Hospital, Kathmandu, Nepal.

\begin{abstract}
Background: Golden proportion, recurrent esthetic dental proportion and golden percentage are the widely accepted tools to measure perceived mesiodistal width of maxillary anterior teeth. Our study was aimed to identify appropriate tool among the above mentioned proportion to measure perceived mesiodistal width.

Methods: A cross-sectional observational study was conducted in National Academy of Medical Sciences, Bir Hospital, Nepalese Army Institute of Medical Sciences and Patan Academy of Health Sciences between March 2014 to March 2015.Sixty-three respondents were recruited in the study by the convenient sampling technique. Photographs of the maxillary anterior teeth were taken using digital single reflex camera with macrolens. Data analysis was done using SPSS version 17.0 and paired sample t- test was performed.

Results: In our study the golden proportion was $14.28 \%$ between central and lateral incisor and $12.69 \%$ between canine and lateral incisor. Therefore, golden proportion in our scenario doesn't exist. Recurrent esthetic dental proportion in female Aryans was 71 and 71 on left side and 71 and 75 on right side. Golden percentage of respondents was near to values $11,15,22,22,15$, and $12 \%$.

Conclusions: Golden percentage is an appropriate anterior tooth proportion which may serve as a guideline for the restoration of anterior tooth. RED proportion is applicable only in Mongoloid female population.

Keywords: Anterior teeth; golden percentage; golden proportion; maxillary; recurrent esthetic dental proportion.
\end{abstract}

\section{INTRODUCTION}

Creating harmonious proportion while restoring or replacing maxillary anterior teeth is difficult task in aesthetic dentistry. ${ }^{1-3}$ If they are not in harmony, psychological and social problems might arise. ${ }^{4-6}$ Golden Proportion(GP) has often been offered as cornerstone of smile design theory. ${ }^{5,7,8}$ Lombardi suggested the application of GP in dentistry through his comprehensive article on esthetics. ${ }^{9}$ The Golden Proportion mathematically denotes ratio of smaller to a larger. ${ }^{10,11}$ Ward suggested Recurring Esthetic Dental (RED) proportion as the proportion of successive width of teeth remaining constant when progressing distally from the midline. ${ }^{6,12}$ Snow recommended Golden percentage as the bilateral analysis of the width of six anterior teeth. ${ }^{3}$ In context of Nepal Rokaya et al. had conducted similar study and concluded that GP cannot be utilized and proposed the new proportion from their study as "Nepalese esthetic dental proportion". ${ }^{13}$

Our study was conducted to identify the appropriate proportion among globally utilized proportions (GP, RED and golden percentage) and use the identified proportion as guide while restoration of maxillary anterior teeth.

\section{METHODS}

A cross sectional study was conducted at National Academy of Medical Sciences (NAMS), Bir Hospital, Nepalese Army Institute of Medical Sciences and Patan Academy of Health Sciences between March 2014 to March 2015.

Based on the study done by Marzok et al.,${ }^{14}$ the sample size was calculated as 63 using the formula of cross sectional 
study. Participants with age ranging between 18-35 years who fulfilled the inclusion criteria were included in the study. Inclusion criteria were: participants without any history of missing tooth, periodontal disease, spacing or crowding in the anterior maxillary teeth during intraoral examination, absence of history of orthodontic treatments, intruded, extruded or rotated teeth in the anterior region.

This study was primarily approved by the Institutional Review Board (IRB) of National Academy of Medical Sciences, which was subsequently endorsed by the IRB of Nepalese Army Institute of Medical Sciences and Patan Academy of Health Sciences. The participants were explained about the aim of study and informed consent was obtained from all the participants prior to their participation. Based on case history and answers obtained through interview, they were categorized primarily into two groups or races as the Aryan and Mongoloid consisting both the male and female. Identified respondents were called at NAMS, Bir Hospital and photographs were taken with a digital single lens reflex camera (Nikon 3100) equipped with a $90 \mathrm{~mm} \mathrm{f/2.8}$ macrolens (Tamaron, Japan) and a macro ring flash. The distance between the camera and the respondents was fixed at a working distance of $30 \mathrm{~cm}(1 \mathrm{~cm}=0.0328 \mathrm{foot})$. The camera was stabilized with the help of a tripod, at this fixed distance. All photographs were taken in same lighting condition and same place for standardization. Respondents were asked to seat comfortably on the chair and instructed to hold their head in natural head position. Frame of the lateral cephalogram machine was used to stabilize head position, for this the earpieces were fitted into the external auditory canals for standardization. In addition, nasion support was placed on the bridge of the nose. Oral retractor was used to displace the soft tissues. Photography standards and rules prescribed by "A Guide to Accreditation Photography produced by the American Academy of Cosmetic Dentistry (AACD Guide)" for upper and lower teeth (frontal and retracted view), was used as a standard for photography. ${ }^{15}$

Photographs were analyzed digitally with software (Adobe Photoshop.CS Version 8). Markings were placed on the most incisal point of the proximal contacts of maxillary anterior teeth; the width of each tooth was computed in pixels. To mark contact points, 5-pixel black dots were used. On all examined digital images, 7 such points were determined; after this, the layer containing the actual picture was deleted, yielding a layer containing only the distances necessary for the measurements.

Data were collected in a spreadsheet (Excel; Microsoft Corp, Redmond, Wash) and analyzed with SPSS 17(IBM Corp, Armonk, NY) and paired T-test was performed. The $p$ values were calculated under the predetermined level of significance (0.05).

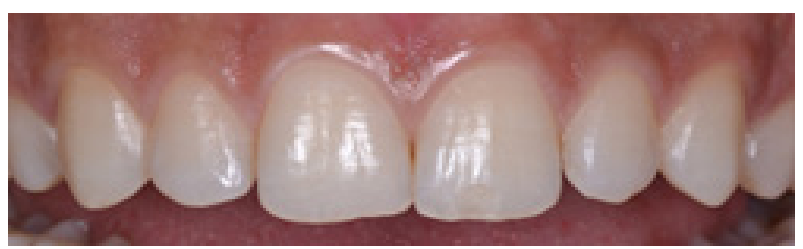

Figure 1. 1:2 ratio retracted frontal view.

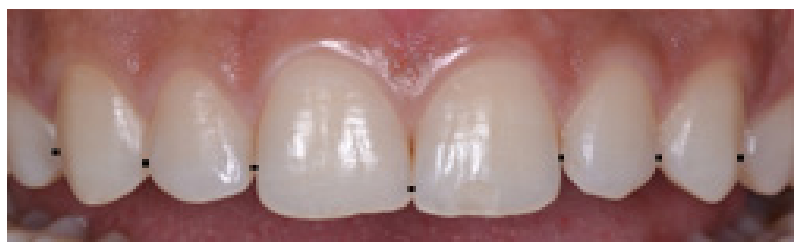

Figure2. 5-pixel black dots on contact. points.

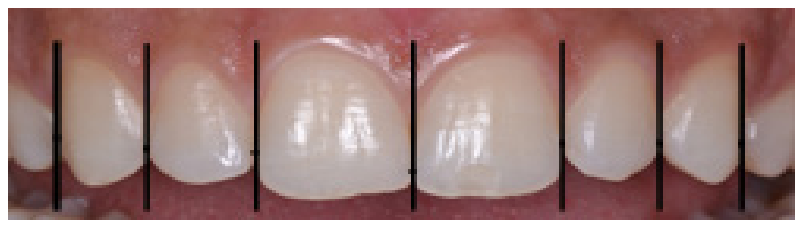

Figure3. Vertical lines (black) on black scale dots for measurement of width.

\section{RESULTS}

The study included total 63 respondents of which $30(47.6 \%)$ were female and $33(52.4 \%)$ were male. Those belonging to Aryan racial group were $33(52.4 \%)$ and Mongoloid were $30(47.6 \%)$

Table 1. Male Aryan and Mongoloid- Golden Percentage.

\begin{tabular}{lccr} 
& N & Mean & Std. Deviation \\
\hline left canine & 18 & 11.54 & 1.52 \\
left Lateral incisor & 18 & 15.65 & 1.23 \\
Left Central incisor & 18 & 22.65 & 1.31 \\
\hline Right Central incisor & 18 & 22.61 & .99 \\
Right Lateral incisor & 18 & 15.42 & .95 \\
Right Canine & 18 & 12.10 & 1.80
\end{tabular}


Clinical Evaluation of Maxillary Anterior Teeth in Relation to Golden Proportion, Red Proportion and Golden Percentage

\begin{tabular}{|c|c|c|c|c|}
\hline \multirow[t]{6}{*}{ Mongoloid } & left canine & 15 & 11.26 & 1.42 \\
\hline & left Lateral incisor & 15 & 15.52 & 1.28 \\
\hline & Left Central incisor & 15 & 22.61 & 1.70 \\
\hline & Right Central incisor & 15 & 22.56 & 1.28 \\
\hline & Right Lateral incisor & 15 & 15.65 & 1.19 \\
\hline & Right Canine & 15 & 12.37 & 1.42 \\
\hline
\end{tabular}

Table 2. Female Aryan and Mongoloid- Golden Percentage.

\begin{tabular}{|c|c|c|c|c|}
\hline \multirow{7}{*}{ Aryan } & & $\mathrm{N}$ & Mean & Std. Deviation \\
\hline & Left canine & 15 & 11.30 & 1.22 \\
\hline & Left Lateral incisor & 15 & 15.95 & .90 \\
\hline & Left Central incisor & 15 & 22.19 & .64 \\
\hline & Right Central incisor & 15 & 22.48 & 1.06 \\
\hline & Right Lateral incisor & 15 & 15.98 & .87 \\
\hline & Right Canine & 15 & 12.06 & 1.11 \\
\hline \multirow{6}{*}{ Mongoloid } & Left canine & 15 & 11.13 & 1.66 \\
\hline & Left Lateral incisor & 15 & 15.86 & 1.93 \\
\hline & Left Central incisor & 15 & 22.72 & 1.06 \\
\hline & Right Central incisor & 15 & 22.88 & 2.19 \\
\hline & Right Lateral incisor & 15 & 15.47 & 1.29 \\
\hline & Right Canine & 15 & 11.9113 & 2.13 \\
\hline
\end{tabular}

Table 3. Male Aryan and Mongoloid- RED Percentage.

\begin{tabular}{llrrrrr} 
& & N & Minimum & Maximum & Mean & Std. Deviation \\
\cline { 2 - 6 } Aryan & Left- Lateral incisor/central incisor & 18 & 57.24 & 87.22 & 69.3921 & 7.35781 \\
\cline { 2 - 7 } & Left -Canine/Lateral incisor & 18 & 52.69 & 96.94 & 74.2520 & 11.75714 \\
\cline { 2 - 7 } & Right- Lateral incisor/Central incisor & 18 & 59.35 & 76.34 & 68.3204 & 5.46281 \\
\cline { 2 - 7 } & Right- Canine/ Lateral incisor & 18 & 50.00 & 102.08 & 78.8567 & 12.89006 \\
\cline { 2 - 6 } & Left- Lateral incisor/central incisor & 15 & 51.57 & 85.22 & 69.0792 & 8.25962 \\
\cline { 2 - 6 } & Left- Canine/Lateral incisor & 15 & 52.63 & 95.65 & 73.2278 & 12.52984 \\
\cline { 2 - 6 } & Right-Lateral incisor/Central incisor & 15 & 55.84 & 78.46 & 69.5144 & 5.62189 \\
\cline { 2 - 6 } & Right- Canine/ Lateral incisor & 15 & 56.64 & 92.45 & 79.2374 & 8.29321 \\
\hline
\end{tabular}

Table 4. Female Aryan and Mongoloid - RED Proportion.

\begin{tabular}{llrrrrr} 
& & N & Minimum & Maximum & Mean & Std. Deviation \\
\cline { 3 - 7 } Aryan & Left- Lateral incisor/central incisor & 15 & 65.12 & 78.81 & 71.8887 & 3.80455 \\
& Left- Canine/Lateral incisor & 15 & 60.18 & 94.06 & 71.2239 & 9.86142 \\
\cline { 2 - 7 } & Right- Lateral incisor/ Central incisor & 15 & 63.77 & 80.00 & 71.1883 & 4.14689 \\
\cline { 2 - 7 } & Right- Canine/ Lateral incisor & 15 & 56.31 & 94.32 & 75.7894 & 9.16157 \\
& Left-Lateral incisor/central incisor & 15 & 60.45 & 103.51 & 70.0437 & 10.64473 \\
\cline { 2 - 7 } & Left- Canine/Lateral incisor & 15 & 56.31 & 106.17 & 71.0323 & 13.50539 \\
& Right- Lateral incisor/Central incisor & 15 & 44.44 & 93.44 & 68.4600 & 10.56629 \\
\cline { 2 - 7 } & Right-Canine/ Lateral incisor & 15 & 56.31 & 110.34 & 77.7757 & 16.88496 \\
\hline
\end{tabular}




\begin{tabular}{ll}
$\begin{array}{l}\text { Table 5. Total percentage of observed Golden } \\
\text { proportion in population. }\end{array}$ & $\begin{array}{l}\text { Canine }=\text { Lateral } \\
\text { Incisor X } 62 \%\end{array}$ \\
$\begin{array}{l}\text { Lateral Incisor }=\text { Central } \\
\text { Incisor X } 62 \%\end{array}$ & $27.77 \%$ \\
\hline $26.19 \%$ &
\end{tabular}

\begin{tabular}{|c|c|c|}
\hline & $\begin{array}{l}\text { Lateral Incisor= Central } \\
\text { Incisor X } 62 \%\end{array}$ & $\begin{array}{l}\text { Canine }=\text { Lateral } \\
\text { Incisor X } 62 \%\end{array}$ \\
\hline Aryan & $13.63 \%$ & $18.18 \%$ \\
\hline Mongoloid & $21 \%$ & $26.66 \%$ \\
\hline
\end{tabular}

\section{DISCUSSION}

The result of the present study revealed the golden proportion was $14.28 \%$ between central and lateral incisor. Golden proportion existed only in $12.69 \%$ between canine and lateral incisor. Therefore, overall result in our scenario showed that the golden proportion does not seem to exist. This was similar to the study conducted by Fayyad et al. ${ }^{16}$ They studied the subjects with esthetic smile and evaluated the existence of golden proportion by measuring the mesio-distal width of six anterior teeth using frontal images of 376 individuals. They concluded that golden proportion did not exist in natural dentition in their context as the golden proportion was found only in $38.2 \%$ between central and lateral incisor and $15.2 \%$ between lateral incisor and canine. ${ }^{16}$ RED proportion as suggested by Ward is the successive width proportion, when viewed from facial aspect, should remain constant as we move posteriorly from the midline. Generally, the values of RED proportion are between $60 \%-70 \% .^{12}$ RED Proportion obtained in the study done by Fayyad et $\mathrm{al}^{16}$ was approximately $70 \%$ and $80 \%$ between central/ lateral incisors and lateral incisor and canine respectively, which was similar for central/lateral incisors but less in case of lateral incisor / canine (70\%) in our study in case of Aryan females. In case of males(Aryan and Mongoloid) RED proportion was obtained approximately $70 \%$ and $80 \%$ between central/ lateral incisors and lateral incisor and canine respectively which was similar to the value obtained in study by Fayyad et al. ${ }^{3}$ but these values don't coincide with the value given by Ward(60-70\%). ${ }^{12}$ Hence, there is significant evidence in this study to support the RED proportion theory as applied to natural dentition of female respondents. However, in case of male respondents, the application of RED proportion was insignificant.

While utilizing the Golden percentage theory to correlate the six anterior teeth, the results of the present investigation suggest that the mean values for golden percentage in Aryan females were 22.1971 - $22.4857 \%$ for central incisors, $15.9541-15.4748 \%$ for lateral incisors and $11.3096-11.9113 \%$ for canine. The values for central incisor, lateral incisor and canine were in near agreement with those suggested by Snow ${ }^{3}$, who recommended the values of 10,15 and 25 as the golden percentage for canine, lateral incisor and central incisor respectively. Similarly, the mean golden percentage for Aryan males were 11.5481, 15.6541, 22.6501 and $22.6178,15.4202,12.1098$ from left canine to right canine respectively whereas for Mongoloid males it was found to be 11.2617, 15.5240, 22.6180, 22.5630, 15.6549 and 12.3784 respectively. The minor variations in the values obtained in this study, as compared to the previous studies, may be attributed to the racial difference of the subjects that were chosen in the present study.

In similar study conducted by Murthy and Ramani ${ }^{17}$ found that out of 56 subjects the golden proportion existed in $17.9 \%$ (left central incisor to left lateral incisor), in 25\% (left lateral incisor to left canines), in $16.1 \%$ (right central incisor to right lateral incisor) and in $14.3 \%$ (right lateral incisor to right canine). But in our study, the golden proportion existed was $14.28 \%$ between central and lateral incisor and $12.69 \%$ between canine and lateral incisor therefore suggesting that the theory of golden proportion was inapplicable in our population.

In the same study conducted by Murthy and Ramani ${ }^{17}$ RED proportions between central incisor and lateral incisor were in the $69.50-70.33 \%$ range and between canine and lateral incisor were in the $80-83 \%$ range. In our study the RED proportion between central incisor and lateral incisor were in the $68.86-70.96 \%$ range and between canine and lateral incisor were in the $71.12-79.02 \%$ range. The RED proportion between central incisor and lateral incisor were found to be similar to the values obtained by Murthy and Ramani. ${ }^{17}$

The values obtained for golden percentage in the study by the Murthy and Ramani ${ }^{17}$ were $12.5,15.5,22,22$, 15.5 and $12.5 \%$, beginning with the right side canine and moving to the left canine respectively, which was found to be similar to our study in case of male and female respondents, i.e. $11.5,15.6,22.65,22.61,15.42$, and $12.1 \%$ and $11.13,15.86,22.72,22.88,15.47,11.91 \%$ respectively.

Similar methodology of measurements was utilized by Foster et $\mathrm{al}^{2}$ in Hungarian population and found that the rule of golden proportions was not valid for the examined 109 participants (36 male, 73 female).The 
ratio of maxillary anterior teeth was 1.6:1:0.85 unlike in our study the ratio which was found to be $1.5: 1: 1.7$ .The canine was markedly wider than predicted by the golden ratio $1.6: 1: 0.6$ in both of the studies. The central incisors were less wide than predicted. No sexdependent or side-dependent variations were found in both studies.

Since the study has small sample size could not represent the Nepalese population, further study with larger sample size is needed.

\section{CONCLUSIONS}

The golden proportion theory is not applicable in our population, the golden percentage is an appropriate anterior tooth proportion which may serve as a guideline in the arrangement of the teeth in edentulous patients and while replacement of anterior teeth in fixed dental prosthesis. RED proportion is applicable only in Mongoloid female population.

\section{REFERENCES}

1. Ali Fayyad M, Jamani KD, Agrabawi J. Geometric and mathematical proportions and their relations to maxillary anterior teeth. J Contemp Dent Pract. 2006;7(5):62-70. [Full Text]

2. Forster A, Velez R, Antal M, Nagy K. Width ratios in the anterior maxillary region in a Hungarian population: addition to the golden proportion debate. J Prosthet Dent. 2013;110(3):211-5. [Link]

3. Snow SR. Esthetic smile analysis of maxillary anterior tooth width: the golden percentage. J Esthet Dent. 1999;11(4):177-84.[Full Text]

4. Gillen RJ, Schwartz RS, Hilton TJ, Evans DB. An analysis of selected normative tooth proportions. Int J Prosthodont. 1994;7(5):410-7.[Link]

5. Mahshid M, Khoshvaghti A, Varshosaz M, Vallaei N. Evaluation of "golden proportion" in individuals with an esthetic smile. J Esthet Restor Dent. 2004;16(3):185-92.[Link]

6. Ward DH. Proportional smile design using the recurring esthetic dental (red) proportion. Dent Clin North Am. 2001;45(1):143-54.[Link]

7. Ettorre G, Weber M, Schaaf H, Lowry JC, Mommaerts MY, Howaldt HP. Standards for digital photography in craniomaxillo-facial surgery - Part I: Basic views and guidelines. J Craniomaxillofac Surg. 2006;34(2):65-73.[Full Text]
8. MA V. Photographs of the face for publication and presentations. J Prosthodont. 2003;12(1):4.

9. Levin EI. Dental esthetics and the golden proportion. J Prosthet Dent. 1978;40(3):244-52.[Science Direct]

10. Paul MMC, Abraham ST. Golden proportion in denture esthetics. Health Sciences. 2013;2(1):10.[Full Text]

11. Lombardi RE. The principles of visual perception and their clinical application to denture esthetics. J Prosthet Dent. 1973;29(4):358-82.[Link]

12. Ward DH. A study of dentists' preferred maxillary anterior tooth width proportions: comparing the recurring esthetic dental proportion to other mathematical and naturally occurring proportions. J Esthet Restor Dent. 2007;19(6):324-37; discussion 38-9. [Link]

13. Rokaya D KJ, Wonglamsam A, Santiwong P, Srithavaj T, Humagain M. Nepalease esthetic dental proportion in Nepalease population. Kathmandu Univ Med J. 2015;13:6.

14. Al-Marzok MI, Majeed KRA, Ibrahim IK. Evaluation of maxillary anterior teeth and their relation to the golden proportion in malaysian population. BMC Oral Health. 2013;13(1):9.[Full Text]

15. Dentistry AAoC. Photographic documentation and Evaluation in Cosmetic Dentistry, A Guide to Accreditation Photography. American Academy of Cosmetic Dentistry. 2009.

16. Fayyad MA, Jamani KD, Aqrabawi J. Geometric and mathematical proportions and their relations to maxillary anterior teeth. JCDP. 2006; 7.[Full Text]

17. Murthi BVS, Ramani N. Evaluation of natural smile: golden proportion, RED or golden percentage. J Conserv Dent. 2008;11. [Link] 